Relations industrielles

Industrial Relations

\title{
Management's Right to Manage, by George W. Torrence. A BNA Operations Manual, The Bureau of National Affairs, Inc., Washington 7, D.C., 1959, 109 pp.
}

\section{Gérard Dion}

Volume 15, numéro 4, octobre 1960

URI : https://id.erudit.org/iderudit/1021952ar

DOI : https://doi.org/10.7202/1021952ar

Aller au sommaire du numéro

Éditeur(s)

Département des relations industrielles de l’Université Laval

ISSN

0034-379X (imprimé)

1703-8138 (numérique)

Découvrir la revue

Citer ce compte rendu

Dion, G. (1960). Compte rendu de [Management's Right to Manage, by George W. Torrence. A BNA Operations Manual, The Bureau of National Affairs, Inc., Washington 7, D.C., 1959, 109 pp.] Relations industrielles / Industrial Relations, 15(4), 514-515. https://doi.org/10.7202/1021952ar

Tous droits réservés (C Département des relations industrielles de l’Université Laval, 1960
Ce document est protégé par la loi sur le droit d'auteur. L'utilisation des services d'Érudit (y compris la reproduction) est assujettie à sa politique d'utilisation que vous pouvez consulter en ligne.

https://apropos.erudit.org/fr/usagers/politique-dutilisation/ 
previously done. It also seems very odd that these « beliefs » and the impossibility of giving the times requested disappear after discharges and he undertook to furnish the required data. It was admitted in evidence that all times which he had previously reported were approximate and it was made abundantly clear that this was the request in this instance. The Board does find that there was insubordination and then proceeds to substitute their judgment for that of Management disregarding the fact that they have no such mandate.

There has been no violation or misinterpretation of any clause and there is no authority for this Board to determine or alter any penalty, therefore, the Board has exceeded its jurisdiction.

\section{RECENSIONS - BOOK REVIEWS}

Organisations ouvrières au Canada, 48e édition, 1959, publié par la Direction de l'économique et de la recherche, Ministère du Travail, Canada, Ottawa, 96 pp.

C'est le nom sous lequel désormais se présente la publication annuelle que nous connaissions sous le titre «Syndicalisme ouvrier au Canada ».

La raison de ce changement est que le répertoire des groupements ouvriers ne se borne plus aux syndicats rattachés à des centrales, mais comprend pour la première fois les organisations locales indépendantes de plus de cinquante membres, qui ont été certifiées comme agent négociateur en vertu de la loi des relations ouvrières appropriée. Pour éviter des susceptibilités, on prend la peine de noter: \& Le fait qu'une organisation paraît dans cette brochure n'implique aucune reconnaissance officielle. Les critères déterminant l'inclusion des organisations dans cette publication n'ont été fixés que dans le but de faciliter l'application de méthodes statistiques cohérentes ».

Comme nos lecteurs connaissent déjà par les éditions antérieures l'utilité de tous les renseignements fournis et l'excellence de cette publication, il nous suffira de remarquer certains changements que nous regrettons. Ainsi, les données statistiques sont moins complètes que par le passé. On ne trouve plus la répartition des unions locales et effectifs par industrie, ni par zône du mar- ché du travail ni par province. De même, dans le répertoire des organisations, ne sont pas indiquées, comme autrefois, les localités où elles possèdent des unités, mais seulement la répartition provinciale. Peut-être, a-t-on des raisons particulières pour n'avoir point fourni ces renseignements, mais c'est dommage. Faut-il aussi souligner que cette édition française nous parvient avec une année de retard?

\section{Gérard Dion}

Management's Right to Manage, by George W. Torrence. A BNA Operations Manual, The Bureau of National Affairs, Inc., Washington 7, D.C., $1959,109 \mathrm{pp}$.

Le but de cette étude est d'examiner ce qui est advenu du droit de gérance que possèdent les dirigeants des entreprises. L'auteur s'adresse aux employeurs afin de leur permettre d'évaluer leur position.

L'ouvrage est divisé en six parties: de quel droit il est question; importance du droit de gérance; ce qui est advenu du droit de gérance, en général; ce qui est advenu du droit de gérance dans des cas particuliers; comment se perd le droit de gérance; comment en arriver à préserver le droit de gérance.

Dans son travail, l'auteur laisse delibérément de côté les restrictions au droit de gérance provenant de la loi ou de la règlementation gouvernementale pour se confiner à celles qui arrivent dans la pra- 
tique des relations patronales-ouvrières et spécialement dans la négociation des conventions collectives.

Toute cette étude est faite dans l'optique patronale, bien que l'auteur admette que le droit de gérance n'est pas absolu ni intangible et que la direction des entreprises soit responsable vis-à-vis des propriétaires, des clients « et enfin des employés ». «So it becomes clear, dit-il, that there are many forces constantly at work which tend to moderate the exercice of management rights. There is one other strong force, too - the ever present knowledge, fortified by history, that the surest way for management to lose its rights (or its job is to abuse them!»

L'auteur donne une bonne nomenclature des différentes situations où les droits de gérance sont impliqués. Et, bien qu'il n'y ait aucun doute du côté où vont ses préférences, dans le but d'éclairer les lecteurs, il fournit objectivement la jurisprudence arbitrale. Après avoir pris connaissance des décisions et des justifications complètement contradictoires apportées par les arbitres dans les cas particuliers où les soi-disant droits de gérance étaient mis en cause, on est porté à croire - ce que ne fait pas l'auteur - que ceux-ci doivent être constamment définis, ou bien assez arbitrairement par l'employeur, ou bien par voíe de négociation.

Les employeurs trouveront dans cet ouvrage tout un arsenal d'arguments pour la négociation de convention collective ainsi que des mises-en-garde pour éclairer leur comportement. Les syndicats ouvriers pourront en tirer profit dans l'établissement de leur stratégie.

\section{GÉrard Dion}

From Field to Factory: New Industrial Employees, par James Sydney Slotkin. Glencoe, Illinois: The Free Press Research Center in Economic Development and Cultural Change, The University of Chicago, 1960, 156 pp., $\$ 4.00$.

Dans ce livre, James Sydney Slotkin, un anthropologue, analyse les problèmes soulevés par l'introduction de l'industrie dans les pays sous-développés où la culture est différente de la culture occidentale. Le problème qui le préoccupe davantage est celui de l'adaptation de la main-d'oeuvre aux nouvelles situations culturelles et sociales, créées par l'industrialisation.

L'auteur tente, à partir de la théorie anthropologique, de construire un modèle général pouvant prévoir toutes les situations possibles. Il veut étendre aux pays industrialisés ce modèle qu'il a développé à partir de l'étude des pays sous-développés. Que le nouveau travailleur industriel vienne d'une culture «primitive » ou d'une région non industrielle d'un pays déjà fortement industrialisé, les processus sont les mêmes. En particulier, le modèle s'appliquerait aux fermiers du Sud qui vont travailler en usine dans la région de Chicago. L'auteur affirme, plus qu'il ne la prouve, cette universalité du modèle. Il cite bien quelques entrevues de fermiers du Sud pour appuyer certains aspects théoriques mais ces citations restent très fragmentaires. On aurait aimé une analyse plus poussée du cas de ces fermiers. Cependant, les exemples fournis laissent croire qu'on puisse accepter, du moins à titre d’hypothèse, cette universalité.

Après avoir défini \&l'industrialisme » comme un système de production dont l'usine de production à la chaîne serait le prototype, il postule que l'industrialisation (I'introduction de cet \&industrialisme 》 dans une société) est un phénomène d'acculturation. Dans cette hypothèse, l'industrialisation sera acceptée d'autant plus facilement que la culture traditionnelle est faible et désorganisée. Ainsi, les travailleurs accepteront de travailler en usine en autant que leur culture sera devenue inadéquate et désorganisée. En particulier, la main-d'oeuvre affluera vers l'usine si la culture iraditionnelle ne permet pas de satisfaire les nouveaux besoins créés par la culture industrielle. On est ainsi dans un processus circulaire. La nouvelle industrie cause une certaine désorganisation de la culture traditionnelle; cette désorganisation poussera les travailleurs vers l'usine, ce qui augmentera la désorganisation, etc.

L'auteur analyse ensuite les problèmes de sélection et d'entraînement des travailleurs. Ces deux fonctions de l'usine seront d'autant facilitées qu'il y aura similitude entre les nouvelles tâches et les occupations traditionnelles des nouveaux travailleurs. Par ailleurs, la rapidité et 